\title{
Ethical issues in conducting placebo controlled clinical trials
}

\section{Opinion}

The placebo-controlled clinical trial has a long history of being the standard for clinical investigations of new drugs. By blindly and randomly allocating similar patients to a control group that receives a placebo and an experimental group, investigators can ensure that any possible placebo effect will be minimized in the final statistical analysis. Although this approach to clinical research is scientifically sound, ethical concerns arise in some cases that outweigh the benefits of this protocol design. ${ }^{1}$ unaccountably, in these times of raised ethical consciousness; placebo treatments are still commonly used in dental research in circumstances in which their use is unethical. Critics of placebo-controlled trials cite article II.3 of the Declaration of Helsinki: "In any medical study, every patient- including those of a control group, if any-should be assured of the best proven diagnostic and therapeutic methods". ${ }^{2}$ Rothman argues that no patient should suffer unnecessary pain, even if the condition being considered is not life-threatening. ${ }^{3}$ It is also unethical in placebo group to withhold treatment and no follow up of participants after the completion of trial. According to the principles of ethics, it is not only the duty of researchers to do no harm but also to do good to the study subjects.

Rothman also argues that placebo-controlled trials are unnecessary and result from a misplaced emphasis on statistical significance testing. ${ }^{3}$ Majority of researchers have a general opinion that studies with statistically significant or positive results are more likely to publish which may allow them to compare a test agent with placebo in the urge of significant results. This could be an unethical issue in conducting placebo controlled trials leading to what are known as "publication bias". ${ }^{4}$ Furthermore, placebo-controlled clinical trials proposed in situations in which effective treatments exist should receive careful scrutiny with regard to whether the effectiveness of the active control is sufficient that interpretable active-control trials could be conducted. Clinical trials that use placebos for indications for which effective or approved medications exist may be perfectly ethical, but close examination of whether patients are adequately informed and whether the trial is consistent with the principles of patient autonomy and physician beneficence is warranted. For the approval of placebo controls in phase 2, 3, and 4 trials, the following questions should be considered: ${ }^{1}$

A. Do participants have a disease or condition for which treatment is available, normally prescribed, and of known efficacy?

B. Will lack of treatment likely result in progression of the disease or condition or the infliction of pain or suffering during the trial?

C. If the disease or condition progresses, is this likely to be reversible?

D. If the disease process is irreversible, how great is the burden of this progression, and how likely is existing treatment to resolve or reduce this burden?
Volume 5 Issue 6 - 2017 Praveen Gadde
Department of Public Health Dentistry Vishnu Dental College,
India

Correspondence: Praveen Gadde, Department of Public Health Dentistry Vishnu Dental College, India, Tel 0779957I724, Email gaddephd6@gmail.com

Received: May 07, 2017 | Published: May 24, 2017

E. Is there substantial evidence that the experimental treatment is of therapeutic benefit?

The answers to these questions should serve to guide institutional review boards in determining whether a placebo- control group is justified. Complete ethical accountability requires that sponsors, institutions, and investigators accept responsibility for subjects who suffer harm because they were given a placebo rather than an available standard therapy. A signed consent form should not shield investigators from claims of malpractice if standard therapy for a condition was intentionally withheld and the subject suffered irreversible harm. Above all, scientific imperatives should never be weighed against established ethical canons.

\section{Acknowledgements}

None.

\section{Conflict of interest}

Author declares that there is no conflict of interest.

\section{References}

1. Chiodo GT, Tolle SW, Bevan L. Placebo-controlled trials: good science or medical neglect? West J Med. 2000;172(4):271-273.

2. World Medical Association Declaration of Helsinki. Recommendations guiding physicians in biomedical research involving human subjects. JAMA. 1997;277(11):925-926.

3. Rothman KJ, Michels KB. The continuing unethical use of placebo controls. N Engl J Med. 1994;331(6):394-398.

4. Hopewell S, Loudon K, Clarke MJ, et al. Publication bias in clinical trials due to statistical significance or direction of trial results. Cochrane Database Syst Rev. 2009;1:1-12. 